\title{
A key requirement for CD300f in innate immune responses of eosinophils in colitis
}

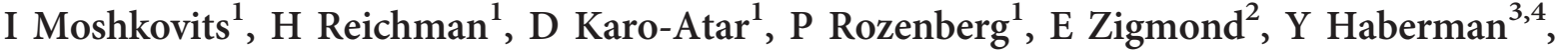

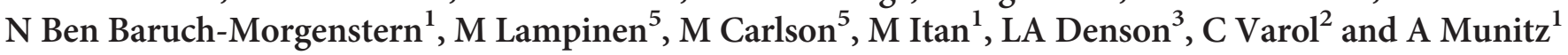

Eosinophils are traditionally studied in the context of type 2 immune responses. However, recent studies highlight key innate immune functions for eosinophils especially in colonic inflammation. Surprisingly, molecular pathways regulating innate immune activities of eosinophil are largely unknown. We have recently shown that the CD300f is highly expressed by colonic eosinophils. Nonetheless, the role of CD300f in governing innate immune eosinophil activities is ill-defined. RNA sequencing of 162 pediatric Crohn's disease patients revealed upregulation of multiple Cd300 family members, which correlated with the presence of severe ulcerations and inflammation. Increased expression of CD300 family receptors was also observed in active ulcerative colitis (UC) and in mice following induction of experimental colitis. Specifically, the expression of CD300f was dynamically regulated in monocytes and eosinophils. Dextran sodium sulfate (DSS)-treated $\mathrm{Cd} 300 \mathrm{f}^{-1-}$ mice exhibit attenuated disease activity and histopathology in comparison with DSS-treated

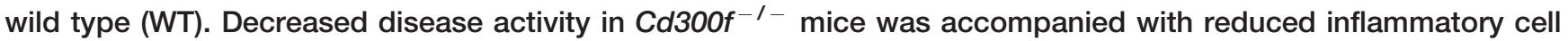
infiltration and nearly abolished production of pro-inflammatory cytokines. Monocyte depletion and chimeric bone marrow transfer experiments revealed a cell-specific requirement for CD300f in innate immune activation of eosinophils. Collectively, we uncover a new pathway regulating innate immune activities of eosinophils, a finding with significant implications in eosinophil-associated gastrointestinal diseases.

\section{INTRODUCTION}

Eosinophils are bone marrow-derived granulocytes that have been traditionally studied in context of type 2 immune responses such as allergy and parasite infections. Yet, recent data highlight new functions for these cells in additional settings such as gastrointestinal (GI) homeostasis and inflammation, tissue regeneration, metabolism, thermogenesis, immune regulation, and cancer. ${ }^{1}$ We have previously demonstrated that during colitis, eosinophils are actively recruited to the inflamed colon by the CCR3-eotaxin pathway, ${ }^{2}$ which is mediated by activation of RelA/p65 in myeloid cells. ${ }^{3,4}$ Eosinophilia was also reported in SAMP1/Yit mice (which develop ileitis resembling Crohn's disease (CD)), ${ }^{5}$ oxazoloneinduced colitis, and in $\mathrm{IllO}^{-/-}$mice, which spontaneously develop chronic colitis. ${ }^{6}$ Conversely, induction of colitis in eosinophil-deficient mice or in mice lacking eosinophil-specific granule proteins results in altered disease phenotype. ${ }^{6,7}$ Indeed, intestinal lamina propria eosinophils express various receptors including Toll-like receptor (TLR)2, TLR4, TLR5, TLR7, and TLR9 that enable them to directly recognize pathogenassociated molecular patterns. Upon TLR stimulation, intestinal lamina propria eosinophils secrete interleukin (IL)-6, which is elevated in colitis. ${ }^{8,9}$ Furthermore, stimulation of IL-5or interferon- $\gamma$-primed eosinophils with lipopolysaccharide initiates the release of mitochondrial DNA in a reactive oxygen species-dependent manner. ${ }^{10}$ Accordingly, we aimed to define molecular pathways that regulate the activities of GI eosinophils with specific emphasis on inhibitory and activating Ig-like receptors, which provide counter regulatory signals for various eosinophil activities. ${ }^{11}$

\footnotetext{
${ }^{1}$ Department of Clinical Microbiology and Immunology, Sackler Faculty of Medicine, Tel Aviv University, Tel Aviv, Israel. ${ }^{2}$ Research Center for Digestive Tract and Liver Diseases, Sourasky Medical Center and Sackler Faculty of Medicine, Tel Aviv University, Tel Aviv, Israel. ${ }^{3}$ Department of Gastroenterology, Hepatology and Nutrition, Cincinnati Children's Hospital Medical Center, Cincinnati, Ohio, USA. ${ }^{4}$ Division of Pediatric Gastroenterology, Hepatology and Nutrition, Edmond and Lily Safra Children's Hospital, Sheba Medical Center, Tel Hashomer, Israel and ${ }^{5}$ Gastroenterology Research Group, Department of Medical Sciences, University Hospital, Uppsala, Sweden. Correspondence: A Munitz (arielm@post.tau.ac.il)
} 
One such family of receptors is the CD300 family. ${ }^{12}$ Most CD300 receptors are considered activating receptors because they possess a short cytoplasmic tail with a charged transmembrane residue that enables their association with adaptor proteins such as DNAX-associated protein (DAP) 12, DAP10, and the $\mathrm{Fc}$ receptor $(\mathrm{FcR}) \gamma$ chain. ${ }^{12}$ In contrast, only $\mathrm{CD} 300 \mathrm{a}$ and CD300f have long cytoplasmic tails with immunoreceptor tyrosine-based inhibitory motifs that enable them to suppress cellular activation. ${ }^{12,13}$ Intriguingly, CD300f has two immunoreceptor tyrosine-based inhibitory motifs, a $\mathrm{p} 85 \alpha$ phosphoinositide 3-kinase (PI3K)-binding motif and a Grb2-binding motif. ${ }^{12-14}$ Thus, CD300f is potentially capable of acting as a co-activating receptor rather than inhibitory. We demonstrated that under homeostatic conditions, colonic CD300f expression is predominantly confined to eosinophils and that CD300f regulated eosinophil homing to the GI tract. ${ }^{15}$ Nevertheless, the contribution of $\mathrm{CD} 300 \mathrm{f}$ to the regulation of eosinophilmediated innate intestinal immune responses remains undefined.

Herein, we demonstrate that the expression of Cd300a, $C d 300 \mathrm{lb}$, and $\mathrm{Cd} 300 \mathrm{f}$ is increased in the ileum of pediatric $\mathrm{CD}$ patients, the colons of active ulcerative colitis (UC) patients, and following induction of experimental colitis in mice. We demonstrate that increased CD300 family member expression in ileal $\mathrm{CD}$ correlates with increased disease severity and expression of $\mathrm{S100a8}$, a hallmark inflammatory marker. Cd300f deficiency protected mice from dextran sodium sulfate (DSS)induced colonic injury that was likely dependent on the expression of CD300f on eosinophils. In fact, direct activation of various $C d 300 f^{-1-}$ cells by $E$. coli led to specific attenuation of pro-inflammatory cytokine production and signaling only in eosinophils. Collectively, these studies emphasize a distinct requirement for $\mathrm{CD} 300 \mathrm{f}$ in the regulation of innate immune eosinophil responses during intestinal inflammation.

\section{RESULTS}

Increased expression of CD300 family receptors in ileal CD patients is associated with mucosal ulcerations and inflammation

To assess the role of CD300 family members in intestinal inflammation, we first analyzed the expression of CD300 molecules in a cohort of patients with early-onset (pediatric) inflammatory bowel disease (IBD), known as the RISK cohort. ${ }^{16}$ Our analysis, which was based on deposited data of $162 \mathrm{CD}$ patients and 42 controls, revealed that the expression of multiple CD300 family receptors was increased in ileal CD patients but not in ileal samples of UC patients, which served as an additional control group ( ${ }^{16}$ and data not shown). These include CD300a, Cd300lb, CD300c, Cd300f, and Cd300e (Figure 1a-e, left panel). CD patients were further stratified according to the presence of deep ulcerations (a marker for disease severity) $)^{16}$ and the expression of CD300 family members was assessed. Expression of all of the identified CD300 family receptors was further increased in patients with deep ulcerations (Figure 1a-e, middle panel). Furthermore, increased expression of CD300 family receptors was associated with increased S100a8 expression, an established marker of gut inflammation (Figure 1a-e, right panel). ${ }^{17}$

To determine whether CD300 family members were increased in the colons of UC patients as well, we accessed deposited genome-wide expression data that were obtained from active UC patients and UC patients in remission. ${ }^{18}$ Interestingly, out of the entire CD300 family of receptors, only the expression CD300f was increased in active UC patients but not in patients in remission (Figure 2a).

These data suggested that the expression of human CD300 family members (and especially of CD300f) were associated with active colonic inflammation. To determine whether the expression of CD300 family receptors was increased in murine colitis, we obtained deposited data that were obtained from temporal genome-wide expression profiling of total colonic tissue in DSS-treated colitis in mice (days 0-6). ${ }^{19}$ This model has been widely studied because it resembles UC and activation of innate immune pathways following colonic injury. ${ }^{2-4}$ Similar to the human data (Figures $\mathbf{1}$ and 2a), DSS treatment induced the expression of numerous murine $\mathrm{CD} 300$ family receptors including Cd300a, Cd300lb, Cd300d, Cd300f, and Cd300g (Figure 2b).

Collectively, our analyses demonstrate that increased expression of Cd300a, Cd300lb, and Cd300f is a shared phenomenon in human and murine intestinal inflammation.

Dynamic regulation of CD300 family receptor expression in monocytes and eosinophils following DSS-induced colitis As CD300a and CD300f are the only CD300 family members containing an intracellular immunoreceptor tyrosine-based inhibitory motif, we next focused on their expression and subsequent function. First, the expression of CD300f and CD300a was determined in lamina propria cells from noninflamed CD patients. ${ }^{20}$ Consistent with our previous findings in murine cells, ${ }^{15}$ human colonic eosinophils expressed the most abundant levels of CD300f in comparison with colonic neutrophils and/or monocytes/macrophages (Figure 2c). In contrast, the expression of CD300a was overall similar in all cell populations with tendency towards higher expression in neutrophils (Figure 2c). Further analysis of CD300a and CD300f expression in circulating eosinophils, neutrophils, and monocytes from control individuals and patients with active and/or quiescent $\mathrm{UC}$ revealed no difference in the expression of CD300a or CD300f between control and UC patients (Supplementary Figure S1 online). Nonetheless, the expression of CD300a and CD300f was substantially increased in lamina propria cells in comparison with peripheral blood cells as can be seen by mean fluorescent intensity values for peripheral blood (Supplementary Figure S1) in comparison with the mean fluorescent intensity of lamina propria cells (Figure 2c).

Next, we assessed the expression of murine CD300a and CD300f in circulating and colonic immune cells following induction of colitis (Figure $\mathbf{2 d - g}$, see gating strategy in Supplementary Figure S2). The expression of CD300f was significantly higher than that of CD300a in the different cell 

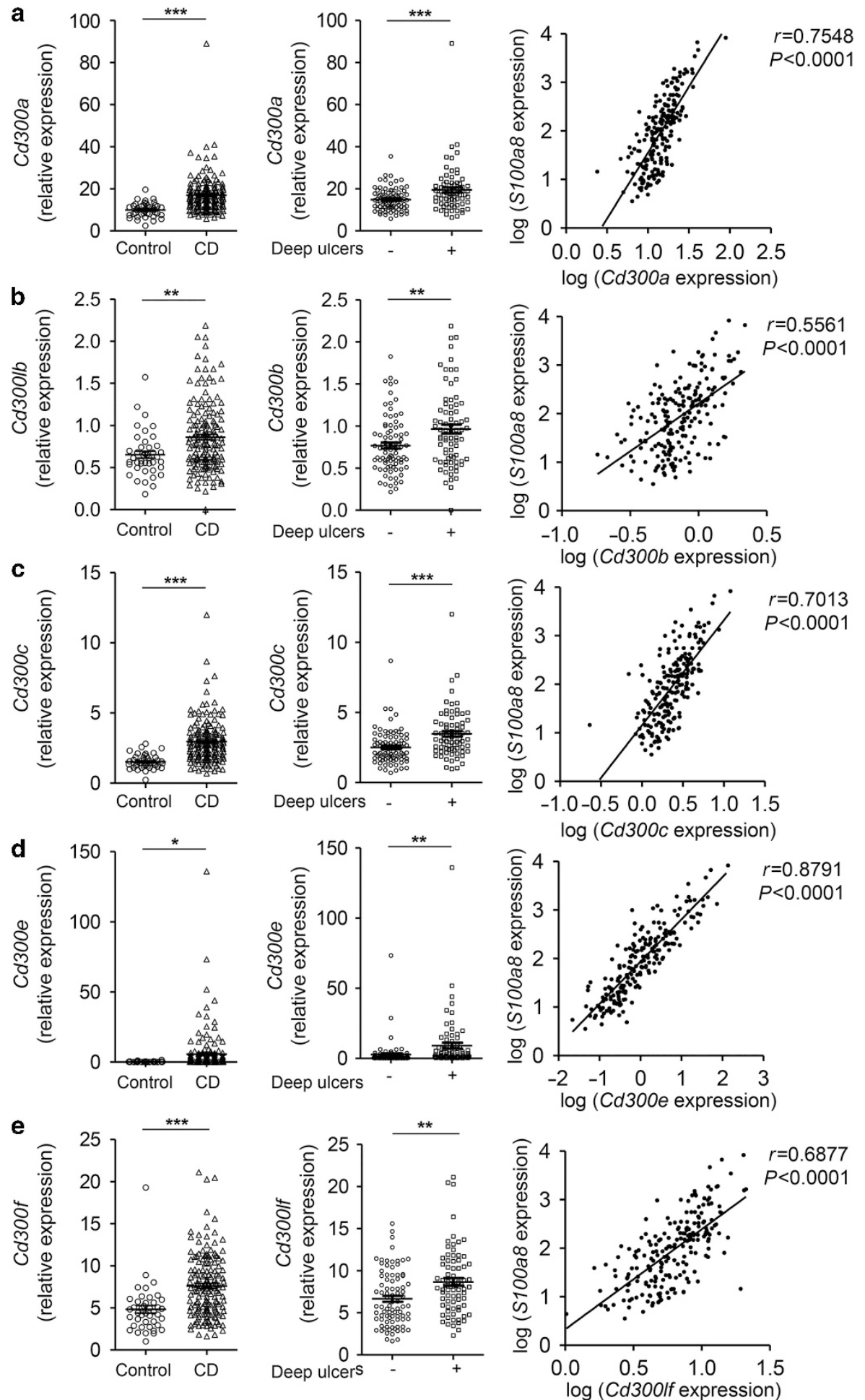

Figure 1 Expression of CD300 family receptors in the ileum of CD patients correlates with deep ulcerations and inflammation. The expression of CD300 family receptors in control and ileal CD patients as determined by RNA sequencing (a-e, left panel). CD patients were stratified accroding to the presence $(+)$ or absence $(-)$ of deep mucosal ulcerations and CD300 family receptor expression was determined (a-e, middle panel). The expression of CD300 family receptors was plotted vs. the expression of the mucosal inflammatory marker s100a8 (a-e, right panel). Data in a-e are from $162 \mathrm{CD}$ patients and 42 control individuals, ${ }^{\star} P<0.05,{ }^{* \star} P<0.01,{ }^{* *} P<0.001$. CD, Crohn's disease.

populations (see the $y$ axis in Figure 2d, $\mathbf{f}$ in comparison with Figure 2e, g). Under baseline conditions, peripheral blood neutrophils expressed the highest levels of CD300f, whereas colonic CD300f was highest in eosinophils (Figure 2d, f). Following DSS treatment, the expression of CD300f in circulating and colonic monocytes was significantly 
a

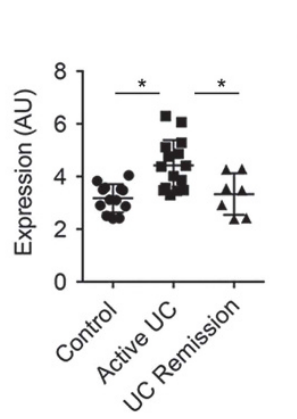

d

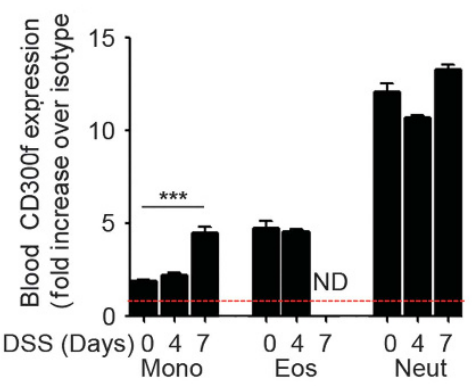

f

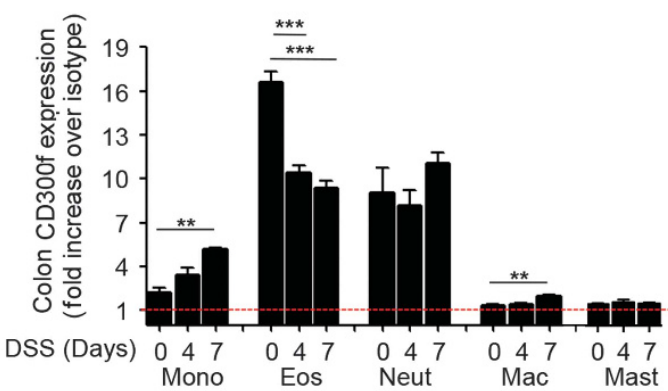

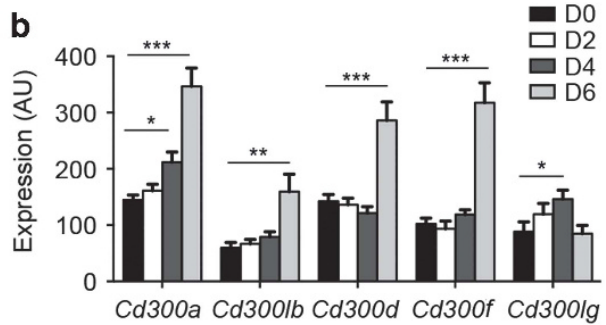

C

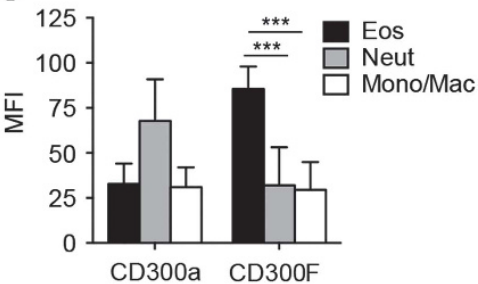

e
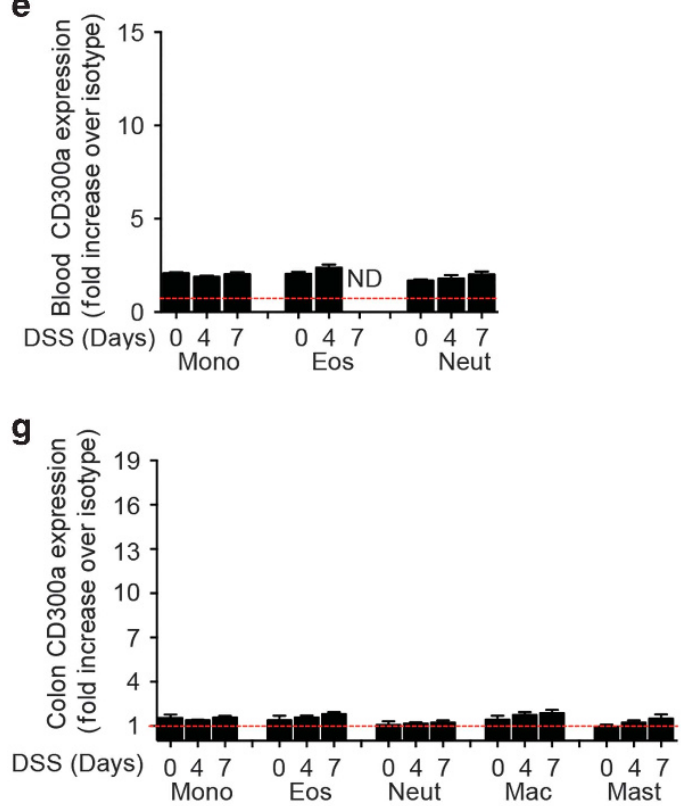

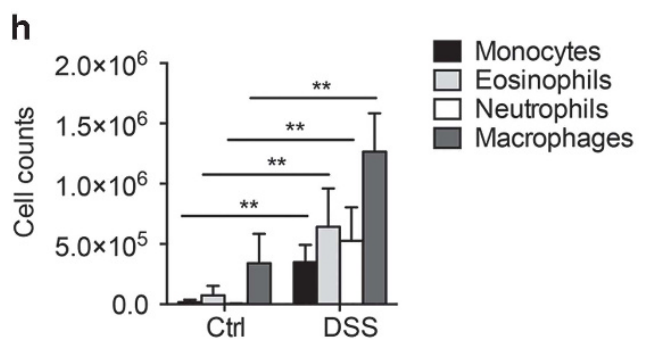

Figure 2 Dynamic regulation of CD300f expression in colitis. Colonic expression of CD300f in control individuals, active UC patients and UC patients in remission is shown (a). Temporal expression of murine CD300 family members in total colonic tissue of DSS-treated mice as determined by analysis of deposited microarray data (Geo Profiles ID: 70456009) (b). Surface expression of human (c) and murine CD300f (d, e) and CD300a (e, g) in peripheral blood $(\mathbf{d}, \mathbf{e})$ and single cell suspensions of colonic lamina propria cells $(\mathbf{c}, \mathbf{f}, \mathbf{g})$. In (b), expression data were obtained from five mice per time point. CD300a and CD300f surface expression in human colonic cells (c) was obtained from nine patients. In (d-g), surface expression of murine CD300a and CD300f is from three to five mice per time point. In (h), total counts of immune cells in the colon are shown. The red line represents the threshold expression level (i.e., the value 1). ${ }^{\star} P<0.05,{ }^{* *} P<0.01,{ }^{* *} P<0.001$. DSS, dextran sodium sulfate; UC, ulcerative colitis.

increased, whereas CD300f expression in colonic eosinophils was decreased and unchanged in neutrophils (Figure 2d, $\mathbf{f}$ and Supplementary Figure S2). The discrepancy between increased CD300f mRNA expression (Figure 2b) and decreased surface expression of CD300f in eosinophils (Figure 2f) is likely explained by the fact that following DSS, the total numbers of $\mathrm{CD} 300 \mathrm{ff}^{+}$cells is increased in the colon rather than increased expression on a single cell basis (Figure 2h). Of note, we could not determine the levels of
CD300f in circulating eosinophils at day 7 because by that time point, peripheral blood eosinophils were completely absent (Figure 2d, e; ND, non determined), likely owing to their recruitment into the inflamed colon. The expression of CD300a was not affected by DSS treatment (Figure 2e, $\mathbf{g}$ ).

\section{CD300f regulates severity of DSS-induced colitis}

Assessment of colitis in $\mathrm{Cd}_{300 f^{-/}}$mice revealed that DSS-treated $C d 300 f^{-/-}$mice displayed attenuated weight loss 

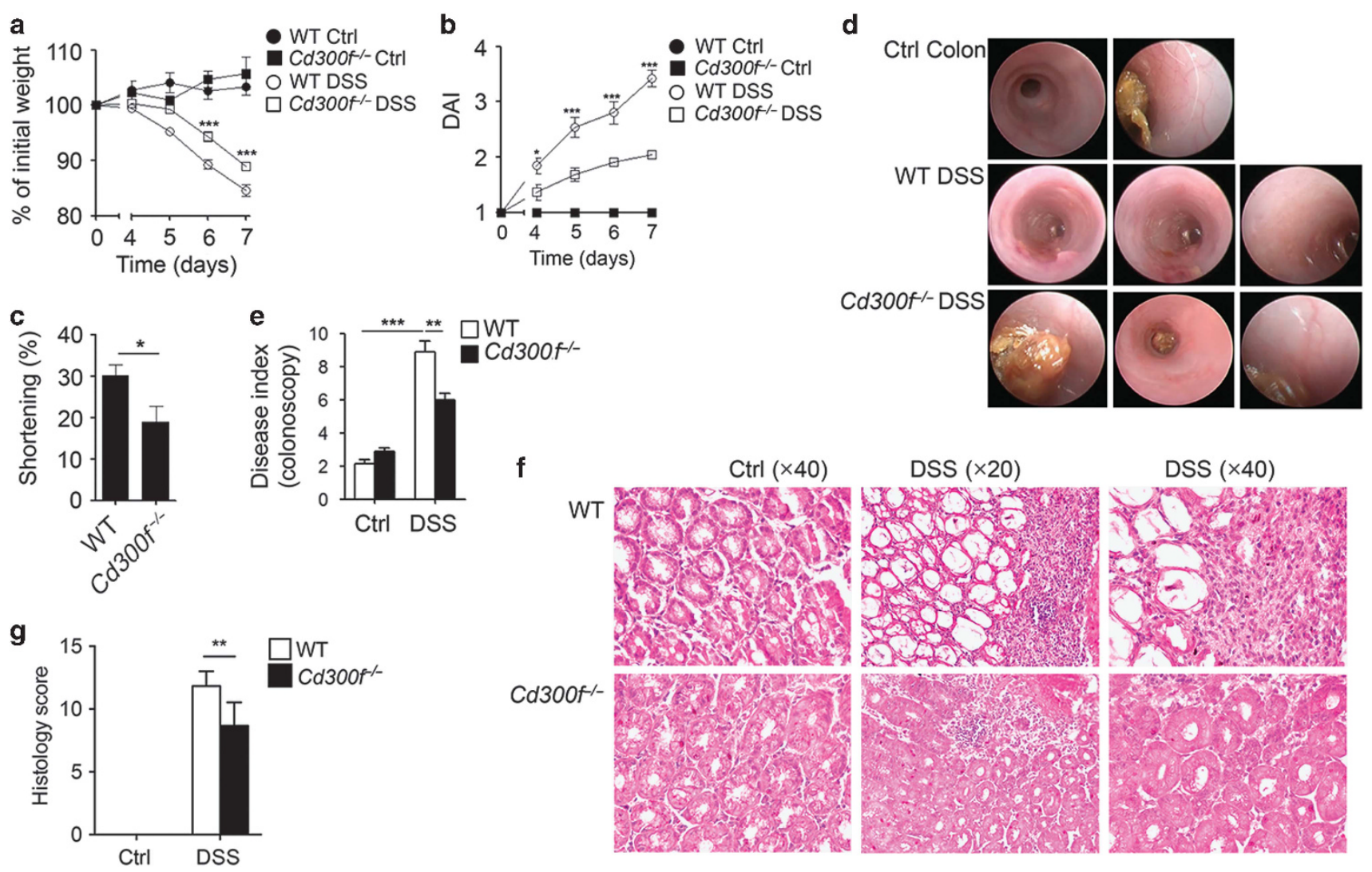

Figure 3 CD300f regulates the severity of of colitis. WT and $C d 300 f^{-/}$mice were subjected to $2.5 \%$ DSS in the drinking water for the indicated time points and analyzed for weight loss (a) and clinical disease activity index (b). The colon length of WT and Cd300f ${ }^{-1-}$ mice following DSS-treatment was assessed using an electronic caliper (c). Representative photomicrographs (d) and colonoscopy disease index (e) comprised of various parameters including stool consistency, rectal bleeding, colon wall transparency, blood vessel architecture, and fibrin deposition are shown (d, e). Representative photomicrographs (f) and histological score $(\mathbf{g})$ of H\&E stained sections from Control (Ctrl) and DSS-treated WT and Cd300f ${ }^{-1-}$ mice is shown. Data are a representative experiment of $n=4$ (6-8 mice per experimental group). ${ }^{\star} P<0.05,{ }^{\star \star} P<0.01,{ }^{\star \star *} P<0.001$. DSS, dextran sodium sulfate; H\&E, hematoxylin and eosin; WT, wild type.

a
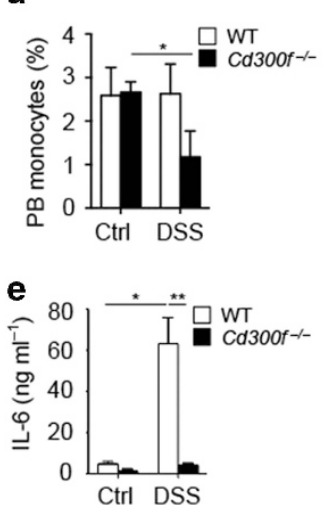

b
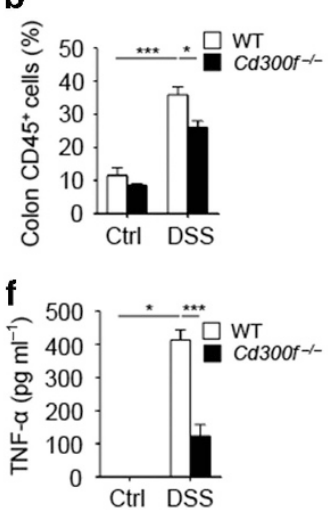
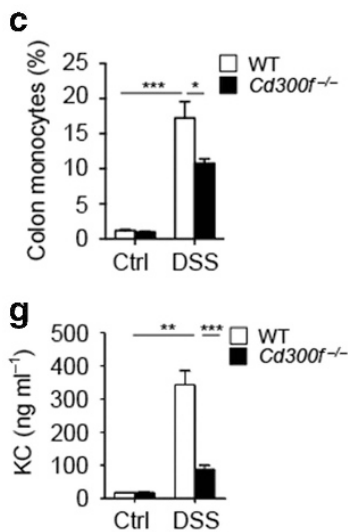
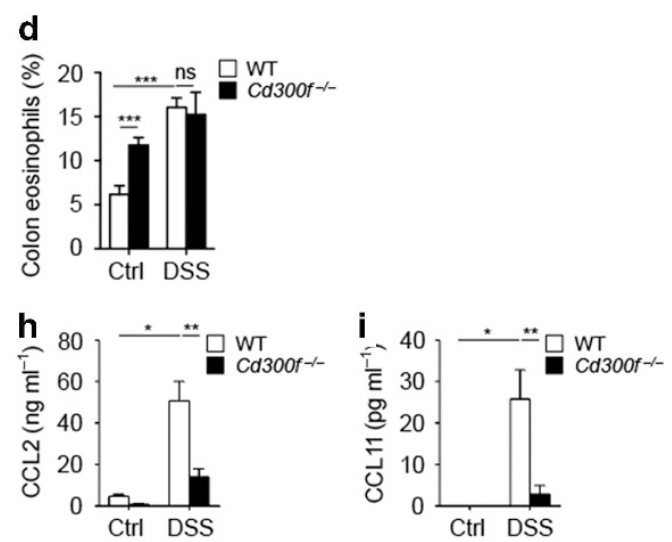

Figure 4 CD300f regulates DSS-induced monocyte infiltration and pro-inflammatory mediator secretion. WT and $C d 300 f^{-1-}$ mice were subjected to $2.5 \%$ DSS in the drinking water for up to 7 days. On day 7 , the levels of peripheral blood monocytes (a), colonic CD45-positive cells (b), colonic monocytes (c), and colonic eosinophils (d) were determined using flow cytometric analysis. IL-6 (e), TNF- $\alpha$ (f), KC (g), CCL2 (h), and CCL11 (i) levels in culture supernatants from distal colon punch biopsies are shown. Data are from $n=4$ experiments (8-10 mice per group). Saline-treated control mice, Ctrl, ${ }^{\star} P<0.05 ;{ }^{* \star} P<0.01,{ }^{\star \star \star} P<0.001$. DSS, dextran sodium sulfate; IL, interleukin; ns, non-significant; TNF, tumor necrosis factor; WT, wild type.

(Figure 3a), rectal bleeding, and diarrhea resulting in decreased disease activity index in comparison with DSS-treated wild-type (WT) mice (Figure 3b). Furthermore, by day 7, DSS-treated WT mice displayed $\sim 30 \%$ shortening of the colon, whereas
DSS-treated $C d 300 f^{-1-}$ mice displayed $\sim 18-20 \%$ shortening (hence a 30\% improvement) (Figure 3c). Colonoscopy examination of DSS-treated WT and $C d 300 f^{-1-}$ mice at day 7 confirmed significantly attenuated colitis score in 
Cd3000f $f^{-1-}$ mice (Figure 3d, e). In contrast to DSS-treated WT mice, DSS-treated $C d 300 f^{-1-}$ mice had visible stool and displayed decreased luminal thickening. Furthermore, they did not lose colonic wall transparency, blood vessel architecture, and displayed less ulcerations (Figure 3d). Finally, histological analysis of colon sections obtained from control and DSS-treated $C d 300 f^{-1-}$ and WT mice revealed no baseline difference between WT and Cd300f ${ }^{-1-}$ mice. Yet, following DSStreatment, $C d 300 f^{-1-}$ mice displayed less erosion and damage of the epithelial layer as well as decreased edema, inflammation, and ulcerations in comparison with WT mice (Figure 3f, g). Genetic deletion of Cd300a had no effect on DSSinduced disease progression (Supplementary Figure S3).

\section{CD300f is required for infiltration of monocytes and expression of pro-inflammatory cytokines following DSS-induced colitis}

Assessment of circulating and colonic inflammatory cells revealed that by day 7, DSS-treated $C d 300 f^{-1-}$ mice had a $50 \%$ decrease in peripheral blood monocyte levels (Figure 4a, \% of $\mathrm{CD} 45^{+}$cells). Furthermore, DSS-treated $C d 300 f^{-/-}$mice had significantly less infiltrating $\mathrm{CD} 45^{+}$cells (Figure $4 \mathbf{b}$ ). In fact, flow cytometric analysis revealed decreased levels of monocytes in the colon of $C d 300 f^{-1-}$ mice following DSS treatment (Figure 4c, \% of CD $45^{+}$cells and Supplementary Figure S4), whereas the levels of additional cells such as eosinophils (Figure 4d and Supplementary Figure S4), neutrophils, and macrophages were unchanged (Supplementary Figure S4). In agreement with our previous report, baseline eosinophil percentages were higher in the colons of $C d 300 f^{-/-}$mice $^{15}$ (Figure 4d, \% of $\mathrm{CD} 45^{+}$cells). Importantly, alterations in blood or colon cell populations were not due to the developmental defects in bone marrow cells of $C d 300 f^{-/-}$ mice (data not shown).

Furthermore, assessment of pro-inflammatory cytokine and chemokine expression from ex vivo colon "punch" biopsies revealed that the secretion of major pro-inflammatory cytokines and chemokines such as IL-6, tumor necrosis factor (TNF) $-\alpha, \mathrm{KC}, \mathrm{CCL} 2$, and CCL11 were nearly abolished in $C d 300 f^{-1-}$ colon cultures (Figure 4e-i).

\section{Decreased DSS-induced pathology in $\mathrm{Cd}_{300 \mathrm{f}^{-/-}}$mice is independent of CD300f expression in monocytes}

We hypothesized that attenuated disease phenotype in DSStreated $\mathrm{Cd} 300 \mathrm{f}^{-/-}$mice may be due to the deficiency of CD300f in monocytes, which have been attributed with proinflammatory functions. ${ }^{21}$ To examine this hypothesis, CCR2 ${ }^{+}$ Ly6C ${ }^{\text {hi }}$ monocytes were depleted in DSS-treated WT and Cd300f ${ }^{-1-}$ mice using the anti-murine CCR2 MC21 antibody (Figure 5a, b and Zigmond et al. ${ }^{21}$ ). Interestingly, monocyte depletion caused a slight but significant increase in colonic eosinophil levels, a finding that was similar in the colons of WT and $\mathrm{Cd} 300 \mathrm{f}^{-1-}$ mice (Figure 5c).

If the protected phenotype was dependent on the expression of CD300f in monocytes, then depletion of monocytes in $C d 300 f^{-1-}$ mice should result in similar severity of DSSinduced disease as in WT mice. However, monocyte-depleted
$C d 300 f^{-/-}$mice still exhibited decreased weight loss and disease activity index in comparison with that of monocytedepleted WT mice (Figure 5d, e).

\section{Decreased DSS-induced pathology in $\mathrm{Cd}_{300 f^{-/-}}$mice is eosinophil-dependent}

To definitely demonstrate the requirement of CD300f in eosinophils functions, we aimed to adoptively transfer purified eosinophils into $\Delta$ dblGATA mice. Despite our efforts, eosinophils that were generated from bone marrow cultures failed to migrate into the colon following retro-orbital injections (data not shown). Furthermore, adoptive transfer of purified eosinophils from the spleens of $I l^{T g}$ mice into $\Delta$ dblGATA mice resulted in preferential homing of these cells into the peritoneal cavity and jejunum but not the colon (Supplementary Figure S5). To specifically probe for the involvement of eosinophil-expressed CD300f in the impaired response of $C d 300 f^{-/-}$mice to DSS treatment, a mixed bone marrow chimera approach was used. To this end, a 1:1 ratio of bone marrow cells from WT or $C d 300 f^{-/-}$ mice together with $\triangle$ dblGATA (where all cells express CD300f) cells was administered to sub-lethally irradiated WT mice. This approach generates animals that harbor a specific CD300f deficiency only in their eosinophil cell compartment (in mice receiving $\Delta$ dblGATA/Cd300f $f^{-/-}$cells) while other hematopoietic cell populations retain their mixed $C D 300 f^{+/+} /$

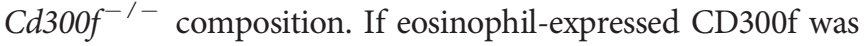
responsible for the protected phenotype that was observed in DSS-treated $C d 300 f^{-1-}$ mice, we would expect to see decreased DSS-induced pathology in mice receiving mixed bone marrow from $\triangle \mathrm{dblGATA} / \mathrm{Cd} 300 \mathrm{f}^{-/-}$in comparison with those receiving mixed $\Delta$ dblGATA/WT cells. Indeed, this experimental regime revealed that WT mice, which received a mixture of $\triangle \mathrm{dblGATA} / \mathrm{Cd} 300 f^{-/-}$bone marrow displayed significantly, decreased disease score and displayed substantially less weight loss in comparison with mice receiving mixed bone marrow cells from $\triangle$ dblGATA/WT mice (Figure 6a, b). In addition, DSS-treated WT mice that received mixed $\Delta$ dblGATA/ WT bone marrow displayed significantly increased shortening of the colon that reached $\sim 45 \%$, whereas mice receiving $\triangle$ dblGATA/Cd $300 f^{-1-}$ bone marrow displayed only a $\sim 30 \%$ reduction in colon length (Figure 6 c, hence a $\sim 30 \%$ improvement). Moreover, the percentage of infiltrating $\mathrm{CD} 45^{+}$ cells was significantly lower in the colons of mice receiving mixed $\triangle \mathrm{dblGATA} / \mathrm{Cd} 300 \mathrm{f}^{-/-}$bone marrow in comparison with mice receiving mixed bone marrow from $\Delta \mathrm{dblGATA} / \mathrm{WT}$ mice (Figure 6d). Finally, histological analysis of colon sections revealed less erosion and damage of the epithelial layer as well as decreased edema, inflammation, and ulcerations in mice receiving mixed $\Delta$ dblGATA/Cd300f $f^{-1}$ bone marrow in comparison with mice receiving mixed bone marrow from $\Delta$ dblGATA/WT mice (Figure 6e).

\section{CD300f is specifically required for innate immune activation of eosinophils}

To corroborate our in vivo findings, the distinct requirement for CD300f in innate immune responses of eosinophils was determined. To this end, we activated various CD300f- 

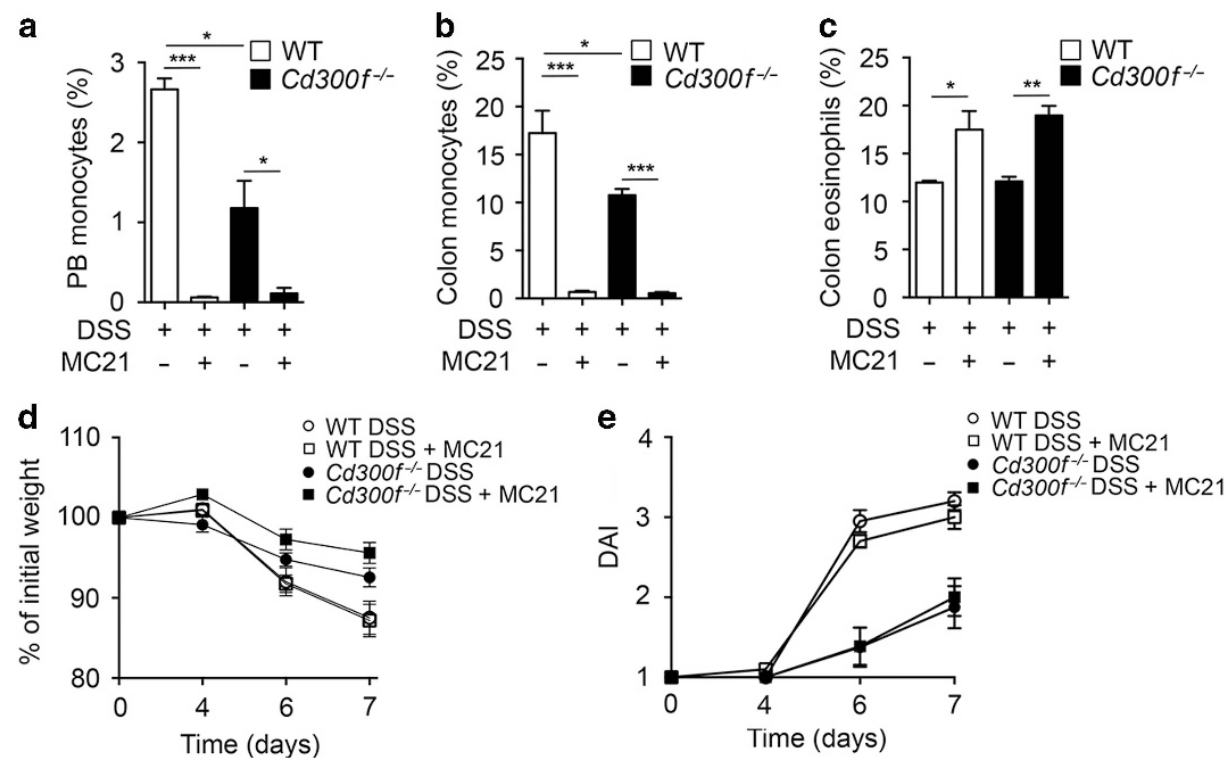

Figure 5 The expression of CD300f in inflammatory monocytes is dispensible for the development of colitis. WT and Cd300f ${ }^{-1-}$ mice were treated with $2 \%$ DSS in their drinking water. To deplete inflammatory monocytes, DSS-treated mice were injected with anti-CCR2 antibody (MC21). The levels of peripheral blood (a) and colonic monocytes (b) as well as colonic eosinophils (c) is shown. DSS-treated and MC21-injected animals were assessed for weight loss (d) and disease activity index (e). Data are from $n=10-11$ mice per group, ${ }^{*} P<0.05 ;{ }^{* \star} P<0.01 ;{ }^{* * *} P<0.001$. DSS, dextran sodium sulfate; WT, wild type.

expressing cells with heat-inactivated E. coli, which was specifically chosen as a representative luminal pathogen, which may be present after breakdown of the luminal wall by DSS. CD300f was specifically required for E. coli-induced IL-6 release from eosinophils but not monocytes, neutrophils, macrophages, and mast cells (Figure 7a-e). Moreover, E. coliinduced TNF- $\alpha$ and $\mathrm{KC}$ secretion was also markedly attenuated in eosinophils (Figure 7f, $\mathbf{g}$ ) but not macrophages or mast cells (Supplementary Figure S6). Importantly, CD300f did not regulate eosinophil-derived IL-4 and IL-13 production, which have been shown to be pathogenic in DSS-induced colitis (Supplementary Figure S7). ${ }^{22,23}$ In support of this, primary colonic eosinophils (but not macrophages) that were sorted from DSS-treated $C d 300 f^{-1-}$ mice displayed a marked decrease in Il6, Ccl24, and Eosinophil Peroxidase (Epx) transcript levels in comparison with eosinophils that were sorted from DSS-treated WT mice (Figure $7 \mathbf{h}-\mathbf{j}$ ).

Finally, assessment of E. coli-induced pro-inflammatory signaling cascades in WT and $C d 300 f^{-1-}$ eosinophils revealed that E. coli-activated $C d 300 f^{-1-}$ eosinophils displayed decreased phosphorylation of JNK, p65 (NFKB), and p38 (Figure 7k-m). Collectively, these data demonstrate a key requirement for $\mathrm{CD} 300 \mathrm{f}$ in innate immune activities of eosinophils.

\section{DISCUSSION}

Innate immune responses of eosinophils are tightly regulated by an intricate network of positive and negative signals. These signals provide a fundamental basis for immune cell homeostasis in health and disease and are thus an attractive therapeutic target in multiple inflammatory diseases. ${ }^{11}$ The CD300 family of receptors draw much attention owing to the fact that their genetic loci is one of only a few loci in the genome that is under strong positive evolutionary selection ${ }^{24}$ likely indicating the importance of these receptors in immune regulation.

Herein, we provide several lines of evidence for a central and distinct role for $\mathrm{CD} 300 \mathrm{f}$ in regulating innate immune responses and subsequently inflammatory signaling in eosinophils during colitis. We demonstrate that the expression of multiple CD300 family receptors is increased in the colon of active UC and in the inflamed ileum of $\mathrm{CD}$ patients. We establish that increased CD300 family receptor expression is correlated with disease severity and inflammatory status of these patients. Increased expression of CD300 family receptors in colonic inflammation is a global and an interspecies phenomenon because increased expression of CD300a, CD300lb, and CD300f was also observed in acute murine colitis. We further demonstrate that the expression of $\mathrm{CD} 300 \mathrm{f}$ is dynamically regulated on the surface of monocytes and eosinophils and that CD300f (but not CD300a) is required for induction of DSS-induced colitis. Notably, chimeric bone marrow experiments suggest that the impact of CD300f in colitis severity is due to the expression of CD300f in eosinophils. Consistently, we demonstrate that CD300f is required for pro-inflammatory signaling and cytokine release specifically from eosinophils in response to innate immune triggers. Collectively, our data highlight a key and novel role for CD300f in inflammatory eosinophil responses during acute colonic inflammation.

Recent data link CD300 family members with intestinal inflammation. ${ }^{12,25}$ Supporting these findings, we demonstrate elevated expression of multiple CD300 family members in biopsies of ileal CD patients and active UC patients. Interestingly, a previous study has assessed the role of CD300f in colitis. ${ }^{26}$ Comparison of those results with our findings yielded 
a

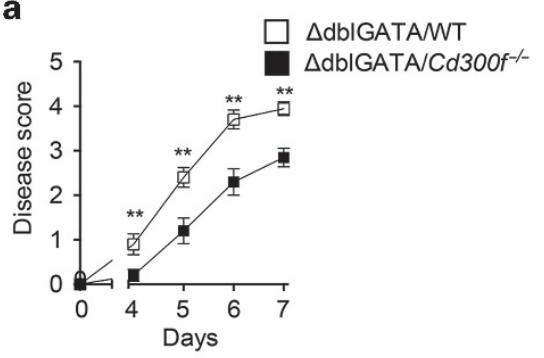

c

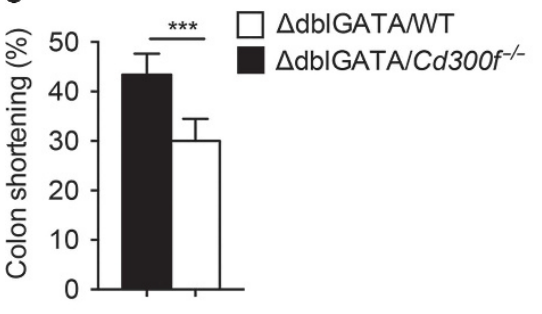

e $\quad \triangle \mathrm{dbIGATANT} \quad \times 10$ b

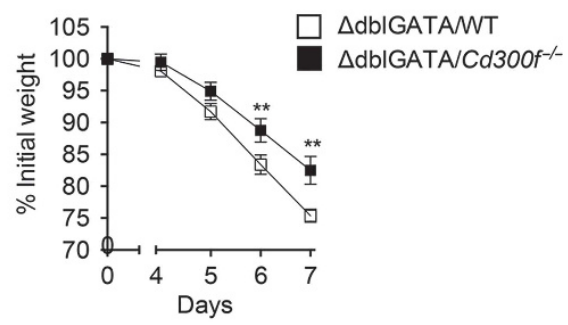

d

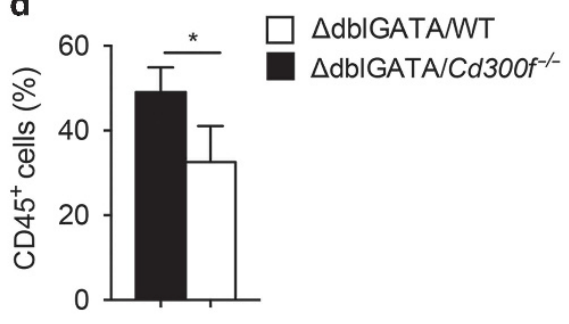

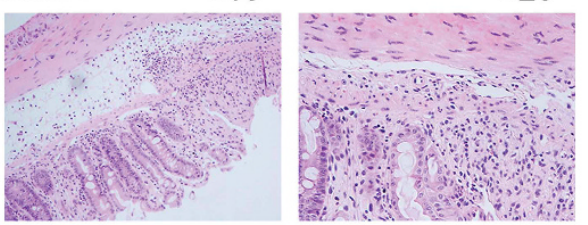

$\triangle \mathrm{dbIGATA} / \mathrm{Cd} 300 \mathrm{f}^{-/-}$
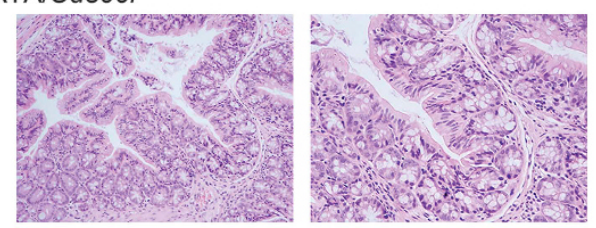

Figure 6 Eosinophil-expressed CD300f regulates the severity of colitis. Mixed BM chimeric mice that harbor a specific deletion of $C d 300 f$ only in their

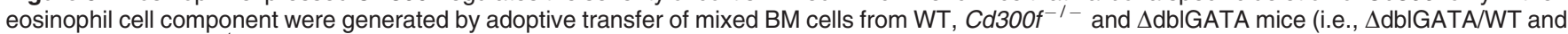
$\triangle \mathrm{dblGATA} / \mathrm{Cd} 300 \mathrm{f}^{-1-}$ ). 0 weeks, the mice were treated with $2.5 \%$ DSS in their drinking water for the indicated time points and analyzed for clinical disease activity index (a), weight loss (b), assessment of colon length (c), and infiltration of CD45 ${ }^{+}$cells into the colon (d). Representative photomicrographs (e) of H\&E-stained sections are shown. Data are a representative experiment of $n=10 ;{ }^{* * *} P<0.001,{ }^{* *} P<0.01$, ${ }^{*} P<0.05$. BM, bone marrow; DSS, dextran sodium sulfate; H\&E, hematoxylin and eosin; WT, wild type.

different conclusions. Whereas our data suggest a requirement for CD300f in innate immune activities of eosinophils, an inhibitory function for CD300f was previously reported. ${ }^{26}$ The latter finding was dependent on the binding of ceramide to CD300f on the surface of mast cells ultimately leading to the suppression of ATP-induced mast cell activities. Potential factors that explain these conflicting results may include technical aspects regarding the induction of colitis using DSS and institutional differences (e.g., dietary source and composition, age of mice) and variability in Cd300f-deficient mouse strains. For example, in our experimental settings, the expression of CD300f was nearly absent in resident colonic macrophages and mast cells, whereas Matsukawa et al. ${ }^{26}$ have shown that under their housing conditions, CD300f was readily detectable in these cells. As the expression of CD300f (and additional CD300 family members) is regulated by various environmental factors including cytokines and innate immune components, $^{27-29}$ this difference suggests that exposure to environmental endotoxins and/or different microbiota communities that are present in the resident animal facilities may be key issues modulating the immune/inflammatory responses linked with CD300f or CD300a. ${ }^{30}$ Furthermore, the dietary availability of lipid mediators (which may influence potential CD300f ligand activities) in different mouse chow may have a critical role in promoting inflammatory responses or supporting resolution mediated by CD300f. ${ }^{31,32}$ Hence, it is possible that each, or any combination, of these factors may be sufficient to alter the intracellular signaling events that are delivered by CD300f. Alternatively, optimal CD300f signaling is obtained by generation of hetero-complexes with additional CD300 receptors. ${ }^{33}$ Thus, it is possible that differential expression of other CD300 family members may affect CD300f signaling. Subsequently, conclusions from both studies may be correct, and the specific circumstances surrounding a given model and protocol will need to be noted in future studies with the multiple $C d 300 f$ mouse models that are currently available. ${ }^{26,34,35}$ 

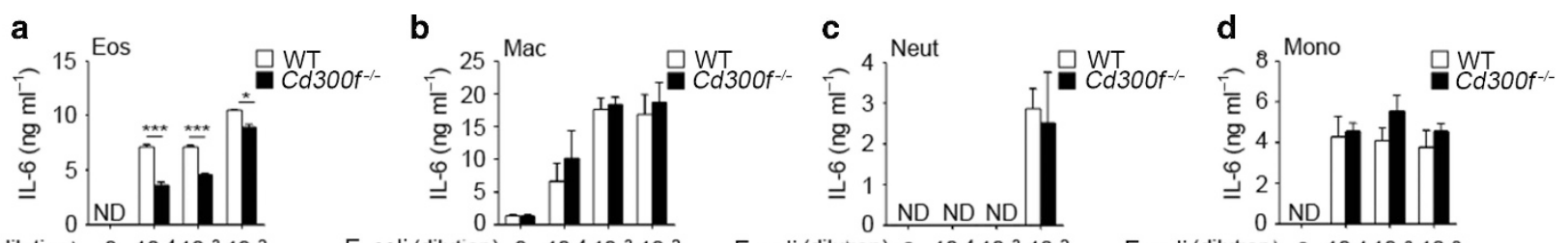

E. coli (dilution) $\quad 0 \quad 10^{-4} 10^{-3} 10^{-2}$

E. coli (dilution) $0 \quad 10^{-4} 10^{-3} 10^{-2}$

E. coli (dilution) $010^{-4} 10^{-3} 10^{-2}$

E. coli (dilution) 0 10-4 10-3 10-2
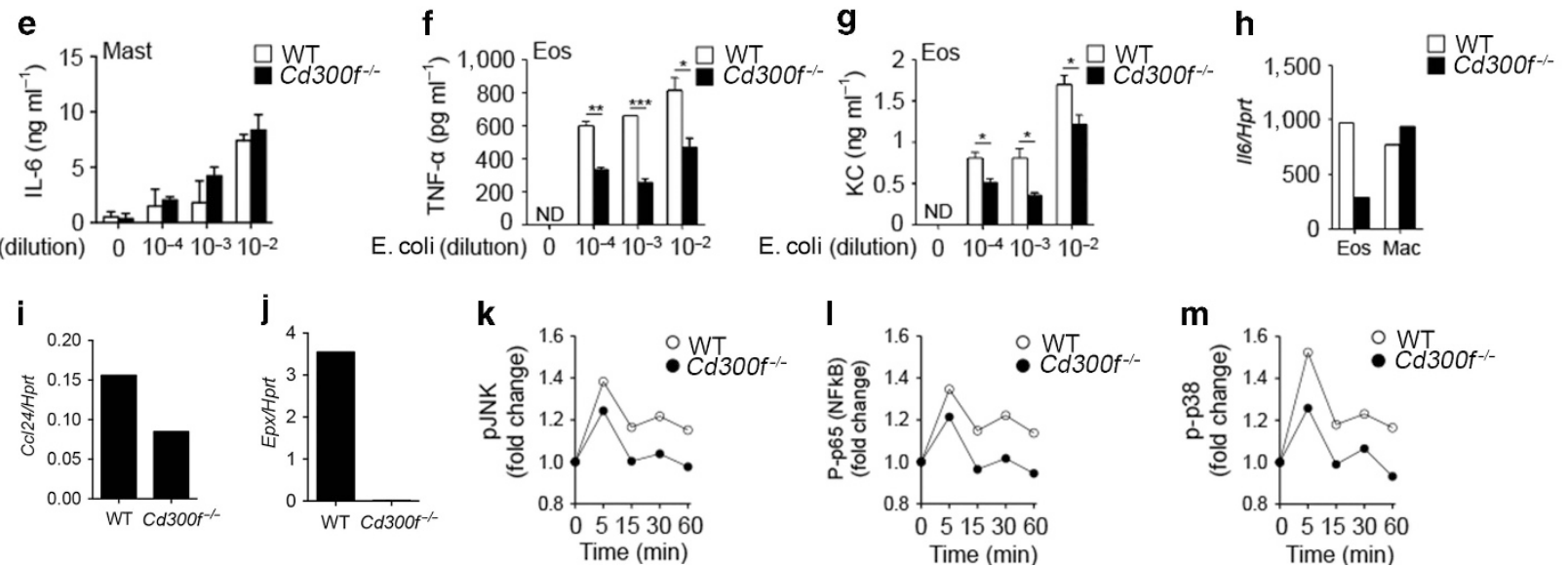

Figure 7 Specific requirement for CD300f in innate immune eosinophil activation. Low density BM-derived eosinophils (Eos, a), BM-derived macrophages (Mac, b), BM neutrophils (Neut, c), and monocytes (Mono, d) as well as BM-derived mast cells (Mast, e) were stimulated with increasing concentrations of heat-inactivated $E$. coli for $24 \mathrm{~h}(\mathbf{a}-\mathbf{e})$. Thereafter, secretion of IL-6 was determined by ELISA (a-e). In addition, secretion of TNF- $\alpha$ (f) and $\mathrm{KC}(\mathbf{g})$ was assessed in the culture supernatants of $E$. coli-stimulated eosinophils. Primary colonic eosinphils and macrophages were sorted from the colons of DSS-treated WT and Cd300f ${ }^{\prime /-}$ mice and transcript expression of $1 / 6, C c l 24$ and Epx were determined (h-j) and normalized to the house keeping gene Hprt. In addition, transcript levels of Ccl24 (i) and Epx (j) were determined in sorted colonic WT and Cd300f eosinophils and normalized to the house keeping gene Hprt. E. coli-induced activation of JNK (k), p65/RelA (I), and p38 (m) was determined by phosphoflow (k-m). Data are representative of $n=3,{ }^{\star} P<0.05 ;{ }^{\star \star} P<0.01 ;{ }^{\star \star \star} P<0.001$; BM, bone marrow; DSS, dextran sodium sulfate; ELISA, enzyme-linked immunosorbent assay; H\&E, hematoxylin and eosin; IL, interleukin; ND, non detected; TNF, tumor necrosis factor; WT, wild type.

Eosinophils have been largely studied in the context of allergic inflammatory diseases. Yet, recent data show that they have key functions in IBD as well. For example, clinical histological analyses describe the involvement of eosinophils in IBD and a sixfold increase in eosinophil levels was reported in biopsy specimens of active UC patients, which was correlated with tissue necrosis. ${ }^{36}$ Ultrastructure studies using electron microscopy revealed the presence of activated eosinophils in established CD patients. ${ }^{37}$ Furthermore, extracellular deposition of eosinophil granule proteins was observed in biopsies, fecal matter, and intraluminal segmental perfusion fluids of IBD patients. ${ }^{38,39}$ The presence of eosinophil granule proteins correlated with morphological changes to the GI tract, increased disease severity, and GI dysfunction. Various independent studies including high-throughput analyses have shown elevated levels of eotaxin (the key eosinophil chemoattractant) in IBD patients, ${ }^{40}$ clarifying the mechanism involved in recruitment of eosinophils into the colon and their localization to substance $\mathrm{P}$ and choline acetyltransferase immunostained nerves. ${ }^{41}$ Previous studies addressed issues regarding the contribution of eosinophils to intestinal inflammation. What remained markedly understudied is the presence of molecular regulators governing eosinophil activities during intestinal inflammation. Thus, our study demonstrating the requirement for $\mathrm{CD} 300 \mathrm{f}$ in innate immune activities of eosinophils is timely and unique.
CD300 family receptors have been shown to bind various ligands that are either expressed by bacteria ${ }^{42}$ or are associated with cell death. ${ }^{12,43}$ In fact, macrophage-expressed CD300 receptors regulate clearance of apoptotic cells via binding of these receptors to phosphatidylserine and alterations in this pathway leads to induction of autoimmunity in $C D 300 f^{-1-}$ mice. ${ }^{35}$ Therefore, we selected the DSS colitis model because it induces epithelial cell shedding and subsequent cell death, ${ }^{44,26}$ and exposes luminal antigens to various immune cells. Thus, the inflamed colon contains multiple ligands for CD300f that are readily available for CD300f-expressing cells such as eosinophils. ${ }^{15,27}$ Interestingly, although the expression of CD300f in eosinophils is decreased following DSS treatment, many CD300f-expressing cells are recruited to the colon following DSS treatment including monocytes, as neutrophils and eosinophils. Thus, we cannot rule out a possibility that CD300f regulates additional functions on non-eosinophil cells (e.g., maturation, migration, or adhesion)

Although no mouse model of colitis model can fully recapitulate all of the pathological processes in human disease, the DSS-induced colitis model is widely used because it reproducibly mimics a model of acute inflammation that is associated with ulcerations. ${ }^{45}$ Directly related, the finding that CD300f is required for the generation of IL- 6 and TNF- $\alpha$, two prominent pro-inflammatory cytokines, is of particular interest. Over the past years, targeting inflammatory cytokines in 
intestinal inflammation has been an emerging therapeutic avenue. Thus, the observation that $\mathrm{CD} 300 \mathrm{f}$ is upstream of proinflammatory signaling and mediator release sets CD300f as a potential anti-inflammatory therapeutic target. We demonstrate a unique requirement for $\mathrm{CD} 300 \mathrm{f}$ in mediating innate immune responses of eosinophils. While we cannot rule out additional roles for CD300f in colitis (especially in human disease), our data suggest that $\mathrm{CD} 300 \mathrm{f}$ is a key receptor governing GI eosinophil activities. Hence, targeting CD300f may be beneficial in IBD patients with increased colonic eosinophilia.

Collectively, our results define a key requirement for CD300f in colonic inflammation likely by the regulation of innate immune eosinophil activation, which is required for induction of pro-inflammatory cell signaling and mediator release. These data highlight CD300f as a potential therapeutic target for suppression of eosinophil functions in multiple eosinophilassociated GI diseases.

\section{METHODS}

Human patients and samples. RNA expression data regarding CD300 family expression and S100a8 expression were obtained from deposited data describing the recently published database describing the specific transcriptome and microbiome signature of ileal CD patients obtained from the RISK cohort. ${ }^{16}$ Additional studies were conducted using patients that were admitted to the Department of Gastroenterology at Uppsala University Hospital, Sweden (seven patients with IBD, four men and three women, mean age 38 years (range 23-53)). Biopsy samples were taken from the sigmoid colon and from ascending colon of all patients. Three of the patients had active inflammation in at least one of the locations, four were in complete remission. The diagnoses were based on established clinical, endoscopic, and histological criteria. ${ }^{46}$ Biopsy samples were taken during colonoscopy (Olympus, Shinjuku, Tokyo, Japan 160 AL endoscope with standard endoscopy forceps) after bowel preparation (i.e., 2 days of diet restriction and an oral purgative in the morning and afternoon of the day before the examination). The procedure does not induce inflammation and is therefore appropriate for this setting. The project was approved by the Ethical Committee of the Medical Faculty, Uppsala University (Approved 8 July 2008), and all patients gave their informed consent to participation in the study.

Mice. The generation of $C d 300 f^{-/-}$mice was previously described. ${ }^{34}$ Cd300a $a^{-/-}$mice (B6N.129S5-Cd300atm1Lex/Mmcd, ID\#032182UCD) were obtained from the Mutant Mouse Regional Resource Center (originally donated by Genentech, San Francisco, CA) and backcrossed to C57BL/6 mice for 10 generations. WT C57BL/6 mice were originally obtained from Harlan Laboratories (Rehovot, Israel) and grown in-house. $I l 5^{T g}$ (NJ.1638) mice were kindly provided by Dr Jamie Lee (Mayo Clinic, Scottsdale, AZ), $\Delta$ dblGATA mice were kindly provided by Dr August Avery (Cornell University, Ithaca, NY). In all experiments, age-, weight-, and sex-matched mice were used and housed under specific pathogen-free conditions according to protocols approved by the Tel-Aviv University Institutional Animal Care Unit.

Bone marrow chimera mice. C57BL/6 (CD45.2) mice were exposed to a single-lethal total body irradiation of $950 \mathrm{rad}$. One day after irradiation, a 1:1 mixture of $5 \times 10^{6}$ bone marrow cells obtained from $\Delta$ dblGATA and WT (CD45.1) or $\Delta$ dblGATA and Cd300f ${ }^{-1-}$ mice and were injected (intravenously) into the irradiated mice, thus generating mixed bone marrow chimeras. Thereafter, the mice were allowed to rest for 10 weeks and engraftment was validated in peripheral blood samples using anti-CD45.1 and anti-CD45.2 staining.
Following confirmation of engraftment, the mice were treated with DSS and disease activity was monitored.

Enzymatic digestion of colonic lamina propria cells. Colonic tissue was excised and flushed with $1 \mathrm{ml}$ of calcium- and magnesium-free HBSS (calcium- and magnesium-free-HBSS). The colon was dissected longitudinally and shacked (250 r.p.m.) in $5 \mathrm{ml}$ calcium- and magnesium-free-HBSS containing $5 \%$ fetal calf serum, $2 \mathrm{mM}$ EDTA, and $1 \mathrm{mM}$ DTT (dithiothreitol) for $40 \mathrm{~min}$ at $37^{\circ} \mathrm{C}$ and in order to remove epithelial cells and intraepithelial lymphocytes. Then, the colonic tissue was vortexed and strained through 70 -micron grey mesh. The remaining tissue was incubated and shacked (250 r.p.m.) with complete phosphate-buffered saline $+/+$ (containing calcium and magnesium) supplemented with $5 \%$ fetal calf serum, $1 \mathrm{mg} \mathrm{ml}^{-1}$ collagenase A (Roche, Berlin, Germany) and $0.1 \mathrm{mg} \mathrm{ml}^{-1}$ Dnase I (Sigma, Rehovot, Israel) for $40 \mathrm{~min}$ at $37^{\circ} \mathrm{C}$. The cell suspension was filtered using gauze (70-micron mesh), washed in incomplete RPMI1640 , and suspended in phosphate-buffered saline.

Flow cytometry. Single-cell suspensions of mouse cells were stained using the following antibodies: anti-CD45-PE-Cy7, anti-CD-45-APC, anti-CD11b-Pe, anti-MHC-II-PE-Cy7, anti-CD11c-Alexa Fluor 488, anti-Gr-1-FITC, anti-Gr-1-PE-Cy7, anti-Ly6C-PerCp-Cy5.5 (obtained from eBioscience, San Diego, CA), anti-CD45-Brilliant Violet 570, rat IgG2a, Armenian hamster IgG (obtained from Biolegend, San Diego, CA), anti-CCR3-FITC, anti-CLM-8 (obtained from R\&D Systems, Minneapolis, MN), anti-Siglec-F-PE (BD Bioscience, San Jose, CA), anti-CD300lf (provided by Dr Menno van Lookeren Campagne, Genentech Inc.), anti-Armenian-hamster-DyLight-649, anti-ratDyLight-649 (Jackson ImmunoResearch, West Grove, PA). For human biopsies staining, mouse anti-human monoclonal antibody conjugated to FITC, PE, PE-Texas Red, PC5, or PC7 were used for CD45, CD15, CD33, and CCR3. Eosinophils were identified as cells double positive for CD15 and CCR3, with lower mean fluorescence intensity (MFI) of CD15 than neutrophils. CD15 ${ }^{\text {high }}$ CCR $3^{-}$cells were gated as neutrophils. Eosinophil and neutrophil identification was confirmed by cytocentrifugation and hematological analyses of sorted $\mathrm{CD} 15^{\text {low }} \mathrm{CCR} 3^{+}$ and CD $155^{\text {high }}$ CCR $3{ }^{-}$cells. Monocytes/macrophages were identified by their forward scatter and side scatter properties and by the expression of CD33 and CD14. Isotype-matched control labeling was also performed, using fluorochrome-conjugated mouse anti-human IgG as a control for nonspecific staining. Antibodies used for flow cytometry were purchased from Beckman Coulter (Bromma, Sweden) and Nordic Biosite (Täby, Sweden). Flow cytometry was performed using either a two-laser FC500 MCL system with CXP or a 3-laser 10-color Gallios flow cytometer (Beckman Coulter, Brea, CA) and data were analyzed using Kaluza (Beckman Coulter) or FlowJo v10.3 (TreeStar, Ashland, OR) analysis software. All staining procedures were conducted following blocking with either $10 \%$ fetal calf serum or using an Fc-blocking reagent (eBioscience). Median fluorescent intensity (MFI) was obtained and $\Delta$ MFI calculated as previously described. ${ }^{15}$

DSS induction of colonic injury. DSS (ICN Biomedical Inc., Santa Ana, CA, average molecular weight of $41 \mathrm{kDA}$ ) was supplied in the drinking water as a $2-2.5 \% \mathrm{w} / \mathrm{v}$ solution for up to 7 days. The appearance of diarrhea was defined as mucus-fecal material adherent to anal fur. The presence or absence of diarrhea was scored as either 1 or 0 , respectively. The presence or absence of diarrhea was confirmed by means of examination of the colon after completion of the experiment. Mice were killed, and the colon was excised from the animal. Diarrhea was defined by the absence of fecal pellet formation in the colon and the presence of continuous fluid fecal material in the colon. The appearance of rectal bleeding was defined as diarrhea containing visible blood, mucus, or both or gross rectal bleeding and scored as described for diarrhea. A change in body weight was calculated by the percent change (gain/loss) from the initial weight. The disease activity index was derived by scoring three major clinical signs (weight loss, diarrhea, and rectal bleeding). 
Intestinal histopathologic examination. Animals were killed on day 7 , and the colon was excised. Tissue specimens were then fixed in $4 \%$ formaldehyde and stained with hematoxylin and eosin using standard histologic techniques.

Punch biopsies. The colons were flushed with phosphate-buffered saline and opened along a longitudinal axis; $3 \mathrm{~mm}^{2}$ punch biopsies were incubated for $24 \mathrm{~h}$ in RPMI supplemented with $10 \%$ fetal calf serum and antibiotics. Supernatants were collected and assessed for cytokine expression.

Colonoscopy. Colonoscopy was performed with a $2.1 \mathrm{~mm}$ miniendoscope (Biovision, Denver, CO). The colonoscope was inserted into the colon up to the splenic flexure $(\sim 3.5 \mathrm{~cm})$ while applying lowlevel air pressure. The white light source was then replaced with a therapeutic light source at the specific tested wavelength, and gradually withdrawn in a predefined rate (which defined the dose) using a laboratory timer. Disease activity scoring was applied as previously described. ${ }^{47}$

Real-time quantitative PCR. cDNA from primary sorted colonic eosinophils and macrophages was subjected to quantitative PCR and normalized to the house keeping gene hypoxanthine-guanine phosphoribosyltransferase (Hprt).

Enzyme-linked immunosorbent assay. Cytokines were measured by enzyme-linked immunosorbent assay according to the manufacturer's instructions (Peprotech, Rehovot, Israel). Lower detection limits for IL-6, TNF- $\alpha$, CCL2, KC, and CCL11 were $62.5,15.6,15.6,15.6$, and $7.8 \mathrm{pg} \mathrm{ml}^{-1}$, respectively.

Monocyte depletion. Mice were treated with anti-CCR2-depleting antibody (M21). Animals were injected with MC21 (5.8 ug per mouse) starting at day 2 after DSS exposure, once a day, for a period of 7 days as described. $^{21}$

Phosphoflow. Bone marrow-derived eosinophils were generated from low-density bone marrow cell, which are enriched with eosinophil progenitor cells. The cells were stimulated with heat-inactivated pathogenic E. coli (ATCC, \#10799) for the indicated time points (0$4 \mathrm{~h}$ ) and phosphoflow analysis was performed as previously described. ${ }^{48}$ The mean fluorescent intensity (MFI) for each intracellular signaling molecule in WT and $C d 300 f^{-/-}$cells at time point 0 min was measured to identify basal phosphorylation levels. Following confirmation of no significant differences in basal MFI levels between groups for each molecule, MFI value for each time point was normalized to baseline and expressed as fold-change over baseline.

Cell culture. Bone marrow-derived eosinophils, bone marrow-derived macrophages, and mast cells were obtained as described elsewhere. ${ }^{28,48-50}$

Statistical analysis. Data were analyzed by analysis of variance followed by Tukey post hoc test, Student's $t$-test, or Pearson productmoment correlation coefficient test using GraphPad Prism 5 (San Diego, CA). Data are presented as mean \pm SD, and values of $P<0.05$ were considered statistically significant.

SUPPLEMENTARY MATERIAL is linked to the online version of the paper at http://www.nature.com/mi

\section{ACKNOWLEDGMENTS}

We wish to thank Dr Moti Gerlic (Tel Aviv University) for critically reviewing this manuscript. AM is supported by the US-Israel Bi-national Science Foundation (grant nos. 2009222 and 2011244), the Israel Science Foundation (grant no. 955/11), the Israel Cancer Research Foundation Research Career Development Award; the Israel Cancer Association (grant no. 20150002), the Israel Ministry of Health (grant no. 3-10117); the Boaz and Varda Dotan Center Grant for Hemato-oncology Research. Itay Moshkovits performed this work in partial fulfillment of the requirements for a PhD degree at the Sackler Faculty of Medicine, Tel-Aviv University, Israel.

\section{AUTHOR CONTRIBUTIONS}

IM, HR, DKA, PR, NBM, LM, EZ, MC, MI, CV, and AM performed experiments; IM, CV, and AM designed the experiments; IM, YH, and LAD analyzed the data; $A M, I M, C V, Y H$, and $L A D$ wrote and edited the manuscript; IM, CV, and A.M. wrote the manuscript.

\section{DISCLOSURE}

The authors declared no conflict of interest.

2017 Society for Mucosal Immunology

\section{REFERENCES}

1. Rosenberg, H.F., Dyer, K.D. \& Foster, P.S. Eosinophils: changing perspectives in health and disease. Nat. Rev. Immunol. 13, 9-22 (2013).

2. Ahrens, R. et al. Intestinal macrophage/epithelial cell-derived CCL11/ eotaxin-1 mediates eosinophil recruitment and function in pediatric ulcerative colitis. J. Immunol. 181, 7390-7399 (2008).

3. Waddell, A. et al. Colonic eosinophilic inflammation in experimental colitis is mediated by Ly6C(high) CCR2(+) inflammatory monocyte/macrophagederived CCL11. J. Immunol. 186, 5993-6003 (2011).

4. Waddell, A. et al. Intestinal CCL11 and eosinophilic inflammation is regulated by myeloid cell-specific RelA/p65 in mice. J. Immunol. 190, 4773-4785 (2013).

5. McNamee, E.N. et al. Novel model of TH2-polarized chronic ileitis: the SAMP1 mouse. Inflamm. Bowel Dis. 16, 743-752 (2010).

6. Furuta, G.T. et al. Eosinophils alter colonic epithelial barrier function: role for major basic protein. Am. J. Physiol. Gastrointest. Liver Physiol. 289, G890-G897 (2005).

7. Forbes, E. et al. Immunopathogenesis of experimental ulcerative colitis is mediated by eosinophil peroxidase. J. Immunol. 172, 56645675 (2004).

8. Chu, V.T. et al. Eosinophils promote generation and maintenance of immunoglobulin-A-expressing plasma cells and contribute to gut immune homeostasis. Immunity 40, 582-593 (2014).

9. Calabrese, L.H. \& Rose-John, S. IL-6 biology: implications for clinical targeting in rheumatic disease. Nat. Rev. Rheumatol. 10, 720-727 (2014).

10. Yousefi, S. et al. Catapult-like release of mitochondrial DNA by eosinophils contributes to antibacterial defense. Nat. Med. 14, 949-953 (2008).

11. Munitz, A. Inhibitory receptors on myeloid cells: new targets for therapy? Pharmacol. Ther. 125, 128-137 (2010).

12. Borrego, F. The CD300 molecules: an emerging family of regulators of the immune system. Blood 121, 1951-1960 (2013).

13. Clark, G.J., Ju, X., Tate, C. \& Hart, D.N. The CD300 family of molecules are evolutionarily significant regulators of leukocyte functions. Trends Immunol. 30, 209-217 (2009).

14. Alvarez-Errico, D., Sayos, J. \& Lopez-Botet, M. The IREM-1 (CD300f) inhibitory receptor associates with the p85alpha subunit of phosphoinositide 3-kinase. J. Immunol. 178, 808-816 (2007).

15. Moshkovits, I. et al. CMRF35-like molecule 1 (CLM-1) regulates eosinophil homeostasis by suppressing cellular chemotaxis. Mucosal Immunol. 7 , 292-303 (2014).

16. Haberman, Y. et al. Pediatric Crohn disease patients exhibit specific ileal transcriptome and microbiome signature. J. Clin. Invest. 124, 3617-3633 (2014).

17. Masoodi, I., Tijjani, B.M., Wani, H., Hassan, N.S., Khan, A.B. \& Hussain, S. Biomarkers in the management of ulcerative colitis: a brief review. Ger. Med. Sci. 9, Doc03 (2011).

18. Planell, N. et al. Transcriptional analysis of the intestinal mucosa of patients with ulcerative colitis in remission reveals lasting epithelial cell alterations. Gut 62, 967-976 (2013).

19. Fang, K. et al. Temporal genomewide expression profiling of DSS colitis reveals novel inflammatory and angiogenesis genes similar to ulcerative colitis. Physiol. Genomics 43, 43-56 (2011).

20. Lampinen, M., Waddell, A., Ahrens, R., Carlson, M. \& Hogan, S.P. CD14 + CD33 + myeloid cell-CCL11-eosinophil signature in ulcerative colitis. J. Leukoc. Biol. 94, 1061-1070 (2013).

21. Zigmond, E. et al. Ly6C hi monocytes in the inflamed colon give rise to proinflammatory effector cells and migratory antigen-presenting cells. Immunity 37, 1076-1090 (2012). 
22. Shajib, M.S. et al. Interleukin 13 and serotonin: linking the immune and endocrine systems in murine models of intestinal inflammation. PloS One 8, e72774 (2013).

23. Pushparaj, P.N. et al. Interleukin-33 exacerbates acute colitis via interleukin-4 in mice. Immunology 140, 70-77 (2013).

24. Bustamante, C.D. et al. Natural selection on protein-coding genes in the human genome. Nature 437, 1153-1157 (2005).

25. Burakoff, R. et al. Blood-based biomarkers can differentiate ulcerative colitis from Crohn's disease and noninflammatory diarrhea. Inflamm. Bowel Dis. 17, 1719-1725 (2011).

26. Matsukawa, T. et al. Ceramide-CD300f binding suppresses experimental colitis by inhibiting ATP-mediated mast cell activation. Gut 65, 777-787 (2016).

27. Shik, D., Moshkovits, I., Karo-Atar, D., Reichman, H. \& Munitz, A. Interleukin-33 requires CMRF35-like molecule-1 expression for induction of myeloid cell activation. Allergy 69, 719-729 (2014).

28. Karo-Atar, D., Itan, M., Pasmanik-Chor, M. \& Munitz, A. MicroRNA profiling reveals opposing expression patterns for miR-511 in alternatively and classically activated macrophages. J. Asthma 52, 1-9 (2014).

29. Moshkovits, I. et al. CD300f associates with IL-4 receptor alpha and amplifies IL-4-induced immune cell responses. Proc. Natl. Acad. Sci. USA 112, 8708-8713 (2015).

30. Nakahashi-Oda, C. et al. Apoptotic epithelial cells control the abundance of T cells at barrier surfaces. Nat. Immunol. 17, 441-450 (2016).

31. Masterson, J.C. et al. Eosinophil-mediated signalling attenuates inflammatory responses in experimental colitis. Gut 64, 1236-1247 (2014).

32. Nickerson, K.P., Chanin, R. \& McDonald, C. Deregulation of intestinal antimicrobial defense by the dietary additive, maltodextrin. Gut Microbes 6 , 78-83 (2015).

33. Martinez-Barriocanal, A., Comas-Casellas, E., Schwartz, S. Jr., Martin, M. \& Sayos, J. CD300 heterocomplexes, a new and family-restricted mechanism for myeloid cell signaling regulation. J. Biol. Chem. 285, 41781-41794 (2010).

34. Xi, H. et al. Negative regulation of autoimmune demyelination by the inhibitory receptor CLM-1. J. Exp. Med. 207, 7-16 (2010).

35. Tian, L. et al. p85alpha recruitment by the CD300f phosphatidylserine receptor mediates apoptotic cell clearance required for autoimmunity suppression. Nat. Commun. 5, 3146 (2014).

36. Bercovitz, Z.T. \& Sommers, S.C. Altered inflammatory reaction in nonspecific ulcerative colitis. Arch. Intern. Med. 117, 504-510 (1966).
37. Dvorak, A.M. Ultrastructural evidence for release of major basic proteincontaining crystalline cores of eosinophil granules in vivo: cytotoxic potential in Crohn's disease. J. Immunol. 125, 460-462 (1980).

38. Saitoh, O. et al. Fecal eosinophil granule-derived proteins reflect disease activity in inflammatory bowel disease. Am. J. Gastroenterol. 94, 35133520 (1999).

39. Sangfelt, P., Carlson, M., Thorn, M., Loof, L. \& Raab, Y. Neutrophil and eosinophil granule proteins as markers of response to local prednisolone treatment in distal ulcerative colitis and proctitis. Am. J. Gastroenterol. 96, 1085-1090 (2001).

40. Mir, A. et al. Elevated serum eotaxin levels in patients with inflammatory bowel disease. Am. J. Gastroenterol. 97, 1452-1457 (2002).

41. Smyth, C.M. et al. Activated eosinophils in association with enteric nerves in inflammatory bowel disease. PloS One 8, e64216 (2013).

42. Phongsisay, V. Campylobacter jejuni targets immunoglobulin-like receptor LMIR5. Mol. Immunol. 63, 574-578 (2015).

43. Simhadri, V.R., Andersen, J.F., Calvo, E., Choi, S.C., Coligan, J.E. \& Borrego, F. Human CD300a binds to phosphatidylethanolamine and phosphatidylserine, and modulates the phagocytosis of dead cells. Blood 119, 2799-2809 (2012).

44. Fischbeck, A. et al. Sphingomyelin induces cathepsin D-mediated apoptosis in intestinal epithelial cells and increases inflammation in DSS colitis. Gut 60, 55-65 (2011).

45. Pizarro, T.T., Arseneau, K.O., Bamias, G. \& Cominelli, F. Mouse models for the study of Crohn's disease. Trends Mol. Med. 9, 218-222 (2003).

46. Sands, B.E. From symptom to diagnosis: clinical distinctions among various forms of intestinal inflammation. Gastroenterology 126, 15181532 (2004).

47. Becker, C., Fantini, M.C. \& Neurath, M.F. High resolution colonoscopy in live mice. Nat. Protoc. 1, 2900-2904 (2006).

48. Baruch-Morgenstern, N.B. et al. Paired immunoglobulin-like receptor A is an intrinsic, self-limiting suppressor of IL-5-induced eosinophil development. Nat. Immunol. 15, 36-44 (2013).

49. Karo-Atar, D., Moshkovits, I., Eickelberg, O., Konigshoff, M. \& Munitz, A. Paired immunoglobulin-like receptor-B inhibits pulmonary fibrosis by suppressing profibrogenic properties of alveolar macrophages. Am. J. Respir. Cell Mol. Biol. 48, 456-464 (2013).

50. Bachelet, I., Munitz, A., Moretta, A., Moretta, L. \& Levi-Schaffer, F. The inhibitory receptor IRp60 (CD300a) is expressed and functional on human mast cells. J. Immunol. 175, 7989-7995 (2005). 